\title{
Efeito da Idade de Lagartas de Spodoptera frugiperda (Smith) (Lepidop- tera: Noctuidae) no Desempenho do Parasitóide Campoletis flavicincta (Ashmead) (Hymenoptera: Ichneumonidae) e Consumo Foliar por Lagartas Parasitadas e Não-Parasitadas
}

\author{
Ivan Cruz ${ }^{1}$, Maria L. C. Figueiredo ${ }^{1}$, Edirlene P. Gonçalves ${ }^{1}$, Dilma A. N. Lima \\ e Elcy E. Diniz ${ }^{1}$
}

${ }^{1}$ EMBRAPA/CNPMS, Caixa postal 151, 35701-970, Sete Lagoas, MG.

An. Soc. Entomol. Brasil 26(2): 229-234 (1997)

Effect of Age of Spodoptera frugiperda (Smith) Larvae (Lepidoptera: Noctuidae) on the Performance of the Parasitoid Campoletis flavicincta (Ashmead) (Hymenoptera:

Ichneumonidae) and Foliar Consumption of Parasitized and Non-Parasitized Larvae

\begin{abstract}
The potential of parasitism of Campoletis flavicincta (Ashmead) (Hymenoptera: Ichneumonidae) on Spodoptera frugiperda (Smith) larvae of different ages and foliar consumption of parasitized and non-parasitized larvae were evaluated. When 3-d old larvae were offered to the parasitoid the number of pararasitized larvae was 232/female, similar to what was obtained with 2-d old larvae (182 larvae/female); with 4-5-d old larvae, parasitism was 80.7 and $71.0 /$ female, respectively. Healthy larvae consumed $209.3 \mathrm{~cm}^{2}$ of leaf, while parasitized larvae consumed $14.5 \mathrm{~cm}^{2}$ (6.9\% of consumption by healthy larvae).
\end{abstract}

KEY WORDS: Insecta, fall armyworm, parasitism, biological control, food intake.

RESUMO - Verificou-se o potencial de parasitismo de Campoletis flavicincta (Ashmead) (Hymenoptera: Ichneumonidae) sobre lagartas de Spodoptera frugiperda (Smith) de diferentes idades e o consumo foliar de lagartas parasitadas, em relação a não parasitadas. Quando foram oferecidas ao parasitóide lagartas de 3-d o número médio de lagartas parasitadas foi de 232/fêmea, número semelhante ao que foi obtido com lagartas de 2-d (182,0 lagartas/fêmea). Com lagartas de 4-5-d, o número médio de parasitismo foi, respectivamente, 80,7 e 71,0 indivíduos parasitados/fêmea. Lagartas sadias, durante todo o seu período de vida, consumiram, em média, $209,3 \mathrm{~cm}^{2}$ de área foliar, enquanto que as lagartas parasitadas consumiram apenas $14,5 \mathrm{~cm}^{2}(6,9 \%$ do consumo de uma lagarta sadia).

PALAVRAS-CHAVE: Insecta, lagarta-do-cartucho, parasitismo, controle biológico, consumo de alimento.

Campoletis flavicincta (Ashmead), parasitóide de lagartas de Spodoptera frugiperda (Smith), tem se mostrado promissor como agente de controle biológico dessa praga da cultura do milho (Zea mays) no Brasil. Em laboratório lagartas de $S$. 
frugiperda de 3-d permitem o desenvolvimento do parasitóide que sai do corpo da lagarta para construir seu casulo externamente (Cruz et al. 1995). Alterações (reduções no tamanho e peso, no número e duração dos instares, pupação e taxa de consumo foliar) causadas por microhimenópteros no desenvolvimento do hospedeiro foram observadas para diferentes insetos como: Heliothis sp., parasitada por Microplitis croceipes (Cresson) (Lewis 1970, Hopper \& King 1984); Heliothis virescens (F.), por Cardiochiles nigriceps Viereck (Vinson 1972); Pseudoplusia includens (Walker), por Meteorus autographae (Muesebeck) (Grant \& Sheppard 1984); S. frugiperda, por Apanteles marginiventris (Cresson), Campoletis grioti (Blanchard), Chelonus insularis (Cresson) e Eiphosoma vitticole Cresson (Ashley 1983), Campoletis sonorensis (Cameron) e Rogas laphygmae Viereck (Isenhour 1988); H. virescens, Spodoptera ornithogalli Guenee (Ables \& Vinson 1981), Trichoplusia ni (Hübner) (Jones et al. 1981); e S. frugiperda (Rezende et al. 1994) por Chelonus sp. O objetivo do trabalho foi verificar o desempenho de $C$. flavicincta em confinamento, sobre lagartas de S. frugiperda de diferentes idades, e avaliar o consumo foliar das lagartas parasitadas em relação a não parasitadas.

\section{Material e Métodos}

Os experimentos foram conduzidos em laboratório, na Embrapa/Centro Nacional de Pesquisa de Milho e Sorgo, Sete Lagoas, MG, em sala climatizada $\left(\mathrm{t}^{\circ}\right.$ de $25 \pm 2{ }^{\circ} \mathrm{C}$, UR de $70 \pm 10 \%$ e fotofase de 12 horas).

Parasitismo e Idade do Hospedeiro. Dezesseis casais recém-nascidos do parasitóide foram individualizados em recipientes de vidro $(9 \mathrm{~cm}$ de diâmetro x 21 cm de altura) com solução açucarada a $5 \%$. Usou-se quatro repetições (cada repetição constituída de quatro casais) cada um recebendo diariamente cerca de 50 lagartas do hospedeiro, com 2 -d de idade ( $1^{\circ}$ instar), 3-d (início do $2^{\circ}$ instar), 4-d (final do $2^{\circ}$ instar) ou 5 -d ( $3^{\circ}$ instar). A cada 24 horas, as lagartas submetidas ao parasitóide foram transferidas para copos de plástico de $50 \mathrm{ml}$, contendo dieta artificial à base de feijão e germe de trigo, e mantidas na mesma sala climatizada. Foram avaliados o número de lagartas parasitadas e o ciclo biológico do parasitóide. Amostras de 10 lagartas de cada idade de $S$. frugiperda foram usadas para se determinar o tamanho do corpo e da cápsula cefálica. Próximo à saída da larva do parasitóide, foram efetuadas diariamente as mesmas medições, para se determinar quando o hospedeiro foi morto pelo parasitóide. O delineamento experimental foi inteiramente casualizado. Os dados sobre comprimento do corpo, largura de cápsula cefálica, número de lagartas parasitadas, períodos biológicos (ovo a pupa, pupa a adulto e ovo a adulto) foram submetidos a análise de variância, e posteriormente ao teste de Duncan $(\mathrm{P} \leq 0,05)$ para comparação de médias.

Consumo Foliar. Lagartas de 3-d obtidas em dieta artificial foram expostas ao parasitismo por $C$. flavicincta por três dias. Para lagartas parasitadas ( $n=34)$, e não-parasitadas $(n=17)$, foram oferecidas folhas de milho (cv. BR 201), medindo-se a cada período de 24-h a área foliar consumida através de um medidor modelo LICOR 300. Pesou-se também as fezes secas. O delineamento experimental foi inteiramente casualizado. Os dados de consumo foliar e peso de fezes foram submetidos a análise de variância, e as médias foram separadas pelo teste de F.

\section{Resultados e Discussão}

Parasitismo e Idade do hospedeiro. As medições realizadas em lagartas parasitadas, indicaram diferenças no comprimento do corpo da lagarta, que variou de 5,41 a 6,06 $\mathrm{mm}$. Entretanto, não houve diferença significativa quanto à largura da cápsula cefálica (média $=0,625 \mathrm{~mm})$ (Tabela 1$)$. De acordo com o número de trocas e pela largura média da cápsula cefálica, a saída da larva do 
Tabela 1. Comprimento do corpo, largura da cápsula cefálica e instar de lagartas de Spodoptera frugiperda antes e após o parasitismo por Campoletis flavicincta.

\begin{tabular}{|c|c|c|c|c|c|c|}
\hline \multirow{2}{*}{$\begin{array}{l}\text { Idade da } \\
\text { lagarta } \\
\text { (dias) }\end{array}$} & \multicolumn{2}{|c|}{$\begin{array}{l}\text { Comprimento do corpo de lagartas de } \\
\text { S. frugiperda }(\mathrm{mm}) \pm \mathrm{EP}^{1}\end{array}$} & \multicolumn{2}{|c|}{$\begin{array}{l}\text { Largura da cápsula cefálica de lagartas } \\
\text { de } S \text {. frugiperda }(\mathrm{mm}) \pm \mathrm{EP}^{1}\end{array}$} & \multicolumn{2}{|c|}{ Ínstar da lagarta } \\
\hline & $\begin{array}{c}\text { Antes do } \\
\text { parasitismo }\end{array}$ & $\begin{array}{c}\text { No dia da } \\
\text { pupação } \\
\text { do parasitóide }\end{array}$ & $\begin{array}{c}\text { Antes do } \\
\text { parasitismo }\end{array}$ & $\begin{array}{c}\text { No dia da } \\
\text { pupação } \\
\text { do parasitóide }\end{array}$ & $\begin{array}{l}\text { Antes do } \\
\text { parasitismo }\end{array}$ & $\begin{array}{c}\text { No dia da } \\
\text { pupação } \\
\text { do parasitóide }\end{array}$ \\
\hline 2 & $1,49 \pm 0,71 \mathrm{c}$ & $6,41 \pm 0,32, a$ & $0,200 \pm 0,00 \mathrm{c}$ & $0,638 \pm 0,03 a$ & 1 & 4 \\
\hline 3 & $2,59 \pm 0,03 b$ & $5,41 \pm 0.12 b$ & $0,300 \pm 0,00 \mathrm{~b}$ & $0,600 \pm 0,00 \mathrm{a}$ & 2 & 4 \\
\hline 4 & $2,56 \pm 0.02 b$ & $6,06 \pm 0,21 \mathrm{ab}$ & $0,300 \pm 0,00 \mathrm{~b}$ & $0,638 \pm 0,02 \mathrm{a}$ & 2 & 4 \\
\hline 5 & $3,60 \pm 0.13 \mathrm{a}$ & $5,99 \pm 0,15 \mathrm{ab}$ & $0,438 \pm 0,02 \mathrm{a}$ & $0,625 \pm 0,02 \mathrm{a}$ & 3 & 4 \\
\hline $\mathrm{CV}(\%)$ & 8,36 & 9,07 & 10,36 & 8,73 & & \\
\hline
\end{tabular}

${ }^{1}$ Médias seguidas pela mesma letra na coluna não diferem significativamente entre si, ao nível de $5 \%$, segundo o teste de Duncan. EP = Erro Padrão da média.

parasitóide ocorreu no $4^{\circ}$ ínstar da lagarta, independente da data do parasitismo.

Para os parâmetros biológicos do parasitóide, embora houvessem diferenças significativas de acordo com a idade do hospedeiro, estas foram inferiores a um período de 1-d (Tabela 2). O período de ovo a pupa do parasitóide variou de 9,2 dias quando foram oferecidas ao parasitóide lagartas de 4-d de idade, a um valor máximo de 10 dias, quando foram oferecidas lagartas de 2-d. Para o período pupal houveram variações de 6,8 a 7,2 dias quando foram oferecidas ao parasitóide lagartas de 3-d e 5d, respectivamente. Já em relação ao ciclo biológico total a variação foi de 16,2 a 16,9

Tabela 2. Número de lagartas de Spodoptera frugiperda parasitadas e duração do ciclo de vida do endoparasitóide Campoletis flavicincta em função da idade do hospedeiro.

\begin{tabular}{|c|c|c|c|c|}
\hline \multirow{2}{*}{$\begin{array}{l}\text { Iddade da } \\
\text { lagarta } \\
\text { (dias) }\end{array}$} & \multirow{2}{*}{$\begin{array}{l}\text { Número de lagartas } \\
\text { parasitadas } \pm \mathrm{EP}^{1}\end{array}$} & \multicolumn{3}{|c|}{ Duração do ciclo de vida } \\
\hline & & $\begin{array}{l}\text { Período de ovo a } \\
\text { pupa (dias) } \pm \mathrm{EP}^{1}\end{array}$ & $\begin{array}{l}\text { Período de pupa a } \\
\text { adulto (dias) } \pm \mathrm{EP}^{1}\end{array}$ & $\begin{array}{l}\text { Período de ovo a } \\
\text { adulto (dias) } \pm \mathrm{EP}^{1}\end{array}$ \\
\hline 2 & $182,0 \pm 44,1 \mathrm{ab}$ & $10,0 \pm 0,3 a$ & $6,9 \pm 0,1 b$ & $16,9 \pm 0,3 a$ \\
\hline 3 & $232,0 \pm 40,6 a$ & $9,5 \pm 0,1 \mathrm{ab}$ & $6,8 \pm 0,1 b$ & $16,3 \pm 0,1 \mathrm{ab}$ \\
\hline 4 & $80,7 \pm 18,4 b c$ & $9,2 \pm 0,1 b$ & $7,0 \pm 0,0 \mathrm{ab}$ & $16,2 \pm 0,2 b$ \\
\hline 5 & $71,0 \pm 23,5 \mathrm{c}$ & $9,6 \pm 0,2 \mathrm{ab}$ & $7,2 \pm 0,1 \mathrm{a}$ & $16,8 \pm 0,2 \mathrm{ab}$ \\
\hline $\mathrm{CV}(\%)$ & 24,5 & 3,8 & 2,8 & 2,4 \\
\hline
\end{tabular}

${ }^{1}$ Médias seguidas pela mesma letra na coluna não diferem significativamente entre si, ao nível de $5 \%$, segundo o teste de Duncan. EP = Erro Padrão da média. 
dias, sendo o menor valor obtido quando foram oferecidas ao parasitóide lagartas de 4-d, e o maior, quando foram oferecidas lagartas de 2-d. Esses valores foram próximos aos obtidos por Cruz et al. (1995) para a mesma espécie e por Isenhour (1986) para a espécie $C$. sonorensis em condições ambientes semelhantes. Entretanto quando os insetos foram criados com folhas de milho, como no experimento visando determinar o consumo foliar de lagartas parasitadas, houve um alongamento no período de ovo a pupa, com média de 21,9 dias (Tabela 3). Além da dieta diferente, o manuseio dos insetos, pela troca constante do substrato alimentar, podem ter sido os fatores responsáveis pela diferença especialmente no período larval tanto do hospedeiro quanto do parasitóide, já que as
80,7 e 71,0 indivíduos parasitados/fêmea, respectivamente. A preferência de fêmeas de C. sonorensis por um instar específico de $S$. frugiperda foi avaliada por Isenhour (1985), que observou ser o $3^{\circ}$ instar de $S$. frugiperda o preferido para o parasitismo, quando comparado ao $2^{\circ}$ e $4^{\circ}$ instares. Por outro lado, o $2^{\circ}$ instar foi o preferido, quando comparado ao $1^{\circ}$. Esses dados diferem em parte dos nossos dados,porque, em termos de lagartas parasitadas, apesar de se ter utilizado um período de parasitismo de apenas 4-h, o maior número foi obtido quando as fêmeas do parasitóide tiveram como hospedeiro lagartas de $2^{\circ}$ instar. Lingren et al. (1970) relataram que $S$. frugiperda foi o melhor hospedeiro, entre sete espécies, para a criação massal de C. sonorensis. Como os autores não

Tabela 3. Área foliar consumida e peso de fezes de lagartas de Spodoptera frugiperda nãoparasitadas e parasitadas por Campoletis flavicincta.

\begin{tabular}{lcccc}
\hline Parâmetro & \multicolumn{2}{c}{ Lagarta Não-Parasitada } & \multicolumn{2}{c}{ Lagarta Parasitada $^{1}$} \\
& Média \pm EP & Amplitude & Média \pm EP & Amplitude \\
\hline Área foliar consumida $\left(\mathrm{cm}^{2}\right)$ & $209,3 \pm 8,7$ & $143,5-269,3$ & $14,5 \pm 1,12$ & $9,7-34,2$ \\
Peso de fezes $(\mathrm{mg})$ & $0,172 \pm 0,007$ & $0,114-0,293$ & $0,007 \pm 0,00$ & $0,003-0,013$ \\
Período de alimentação (dias) & $25,0 \pm 0,6$ & $23-31$ & $14,5 \pm 0,5$ & $12-20$ \\
\hline
\end{tabular}

${ }^{1}$ Médias significativamente diferentes ao nível de $5 \%$, segundo o teste t.

condições ambientes foram semelhantes. $\mathrm{O}$ período pupal de 7,3 dias foi semelhante ao obtido no experimento envolvendo a dieta artificial.

A taxa de parasitismo variou significativamente com a idade do hospedeiro (Tabela 2). O número médio de lagartas parasitadas para cada fêmea foi de 232, quando ao parasitóide foram oferecidas lagartas de3-d de idade; esse valor só não diferiu daquele obtido para lagartas de 2-d de idade, cuja média por fêmea foi de 182,5 indivíduos parasitados. Em lagartas de 4 e 5d, a média de parasitismo foi menor, ou seja, forneceram dados de temperatura, não se pode ter uma estimativa do instar estudado em função da idade. No entanto, concluíram que lagartas de 3-4 d de idade foram as preferidas para a oviposição. Três dias foi a idade em que se verificou a maior taxa de parasitismo de C. flavicincta (Tabela 2).

Poucos são os trabalhos direcionados para se determinar o potencial de parasitismo de Campoletis sobre lagartas de S. frugiperda. Isenhour (1986), testando o parasitóide $C$. sonorensis contra $S$. frugiperda, em condições semelhantes ao presente trabalho de $\mathrm{t}^{\circ} \mathrm{e}$ UR, porém oferecendo ao parasitóide lagartas de 
$3^{\circ}$ instar, encontrou um número médio de 198,8 lagartas parasitadas, valor inferior ao encontrado no presente trabalho, (232 lagartas parasitadas/fêmea) (Tabela 2). Apesar de um aumento na taxa de parasitismo a $30^{\circ} \mathrm{C}(224,5$ indivíduos parasitados/fêmea), o valor foi inferior ao obtido neste trabalho. Aparentemente o desempenho de C. flavicincta é melhor do que $C$. sonorensis sobre lagartas de $S$. frugiperda.

Consumo Foliar. As lagartas sadias de $S$. frugiperda alimentaram-se da folha de milho por um período médio de 25 dias enquanto que as parasitadas, alimentaram-se por um período de 17,5 dias (Tabela 3), ou seja, cerca de uma semana menos. Embora o ciclo larval das lagartas parasitadas não tenha sido diferente do ciclo das lagartas sadias, verificaram-se diferenças relativas ao consumo foliar entre lagartas parasitadas e não parasitadas. Lagartas sadias consumiram, em média, $209,3 \mathrm{~cm}^{2}$ de área foliar, enquanto que as lagartas parasitadas consumiram $14,5 \mathrm{~cm}^{2}$, ou seja, $6,9 \%$ do consumo normal. Esse menor consumo de alimento foi confirmado pela quantidade de fezes produzida (lagartas sadias $=0,172 \mathrm{mg}$, comparada com $0,007 \mathrm{mg}$ produzida pelas lagartas parasitadas $-4,1 \%$ da produção total). Portanto, além de provocar a morte das lagartas, o parasitóide reduz drasticamente o consumo foliar.

A redução no consumo foliar também tem sido verificada quando as lagartas de $S$. frugiperda são parasitadas por Chelonus insularis (Cresson) (Rezende et al. 1994). Esses autores trabalhando em condições semelhantes, verificaram que lagartas parasitadas consumiram um total de $12,2 \mathrm{~cm}^{2}$ de área foliar, cessando sua alimentação no $14^{\circ}$ dia, quando iniciou-se o período de prépupa. Lagartas não parasitadas consumiram $179,7 \mathrm{~cm}^{2}$ de área foliar, alimentando-se até o $18^{\circ}$ dia. C. insularis é um parasitóide que coloca seus ovos no interior dos ovos de $S$. frugiperda (Rezende et al. 1994), enquanto que $C$. flavicincta coloca seus ovos em lagartas pequenas (Cruz et al. 1995). Apesar de se terem valores inferiores para o consumo, tanto por lagartas sadias como por parasitadas por $C$. insularis, a razão entre área foliar consumida por lagartas parasitadas em relação às não parasitadas $(6,8 \%)$ foi muito próxima da razão obtida para $C$. flavicincta $(6,9 \%)$. Diferença maior foi obtida quando se compara o peso das fezes. A produção de fezes por lagartas parasitadas por Chelonus foi de 0,03 $\mathrm{mg}$, enquanto que a de lagartas parasitadas por Campoletis foi de apenas $0,007 \mathrm{mg}$. Apesar dessa diferença, em função de uma maior produção de fezes por lagartas sadias no experimento com Campoletis $(0,172 \mathrm{mg} /$ lagarta), em comparação ao experimento com Chelonus $(0,38 \mathrm{mg} / \mathrm{lagarta})$, a razão entre fezes produzidas por uma lagarta parasitada em relação a uma não-parasitada foi bem diferente, ou seja, 4,1\% para Campoletis e 7,9\% para Chelonus.

Isenhour (1988), comparando o ganho de peso e a quantidade de folhas de milho e de sorgo (Sorghum vulgare) consumida por $S$. frugiperda, que havia sido parasitada tanto por $C$. sonorensis como por R. laphygmae, verificou que as lagartas parasitadas apresentaram peso e quantidade de folha consumida significativamente inferiores em relação a lagartas não-parasitadas. $O$ consumo foliar de lagartas parasitadas (9-d idade) por C. sonorensis, num período de 6-d de alimentação, foi de $4,6 \mathrm{~cm}^{2}$, comparado com o consumo de $17,8 \mathrm{~cm}^{2}$ por lagartas não parasitadas. A habilidade dos inimigos naturais em reduzir o dano infligido à planta os tornam desejáveis como agentes de controle biológico. Um consumo foliar menor por lagartas parasitadas também tem sido verificado em outras plantas com outros insetos. Por exemplo, o consumo foliar de soja por lagartas de $P$. includens parasitadas por M. autographae foi significativamente menor $(\mathrm{P}<0,05)$ do que o consumo por lagartas não parasitadas, respectivamente 13,4 e $61,5 \mathrm{~cm}^{2}$ (Grant \& Sheppard 1984).

O parasitóide $C$. flavicincta tem sido recuperado com freqüência em coletas de lagartas de S. frugiperda a campo. Os resultados apresentados neste trabalho permitem concluir que esse parasitóide possui 
potencial de utilização como agente de controle biológico da lagarta-do-cartucho de milho, no Brasil, dada a sua capacidade de provocar mortalidade em populações da praga e reduzir sua alimentação.

\section{Literatura Citada}

Ables, J.R. \& S.B. Vinson. 1981. Regulation of host larval development by the egglarval endoparasitoid Chelonus insularis (Hymenoptera: Braconidae). Entomophaga 20:453-458.

Ashley, T.R. 1983. Growth pattern alterations in fall armyworm, Spodoptera frugiperda, larvae after parasitization by Apanteles marginiventris, Campoletis grioti, Chelonus insularis and Eiphosoma vitticole. Fla. Entomol. 66: 260-266.

Grant, J.F. \& M. Sheppard. 1984. Laboratory biology of Meteorus autographae (Hymenoptera: Braconidae) an indigenous parasitoid of soybean looper (Lepidoptera: Noctuidae) larvae. Environ. Entomol. 13: 838-842.

Cruz, I., D.A.N. de Lima, M.L.C. Figueiredo \& F.H. Valicente. 1995. Aspectos biológicos do parasitóide Campoletis flavicincta (Ashmead) criados em lagartas de Spodoptera frugiperda (Smith). An. Soc. Entomol. Brasil. 24: 201-208.

Hopper, K.R. \& E.G. King. 1984. Feeding and movement on cotton of Heliothis species (Lepidoptera: Noctuidae) parasitized by Microplitis croceipes (Hymenoptera: Braconidae). Environ. Entomol. 13: 1654-1660.

Isenhour, D.J. 1985. Campoletis sonorensis (Hym.: Ichneumonidae) as a parasitoid of Spodoptera frugiperda (Lepidoptera: Noctuidae): host stage preference and functional response. Entomophaga 30: 31-36.

Isenhour, D.J. 1986. Developmental time, adult reproductive capability, and longevity of Campoletis sonorensis (Hymenoptera: Ichneumonidae) as a parasitoid of fall armyworm, Spodoptera frugiperda (Lepidoptera: Noctuidae). Ann. Entomol. Soc. Am. 79: 893-897.

Isenhour, D.J. 1988. Interactions between two hymenopteous parasitoids of the fall armyworm (Lepidoptera: Noctuidae). Environ. Entomol. 17: 616-620.

Jones, D., G. Jones \& B.D. Hammock. 1981. Developmental and behavioural responses of larval Trichoplusia ni to parasitization by an imported braconid parasite Chelonus sp. Physiol. Entomol. 6: 387-394.

Lewis, W.J. 1970. Study of species and instars of larval Heliothis parasitized by Microplitis croceipes. J. Econ. Entomol. 63: 363-365.

Lingren, P.D., R.J. Guerra, J.W. Nickelsen \& C. White. 1970. Host and host age preference of Campoletis perdistinctus. J. Econ. Entomol. 63: 518-522.

Rezende, M.A.A., I. Cruz, T. M. C. Della Lucia. 1994. Consumo foliar de milho e desenvolvimento de lagartas de Spodoptera frugiperda parasitadas por Chelonus insularis. An. Soc. Entomol. Brasil 23: 473-478.

Vinson, S.B. 1972. Factors involved in successful attack on Heliothis virescens by the parasitoid Cardiochiles nigriceps. J. Invertebr. Pathol. 20: 118-123.

Recebido em 03/06/96. Aceito em 20/05/97. 